\title{
Effective Stability for Periodically Perturbed Hamiltonian Systems
}

\author{
Àngel Jorba, ${ }^{1}$ and Carles Simó ${ }^{2}$ \\ ${ }^{1}$ Departament de Matemàtica Aplicada I, ETSEIB \\ Universitat Politècnica de Catalunya \\ Diagonal 647, 08028 Barcelona (Spain) \\ ${ }^{2}$ Departament de Matemàtica Aplicada i Anàlisi \\ Universitat de Barcelona \\ Gran Via 585, 08007 Barcelona (Spain)
}

\begin{abstract}
In this work we present a method to bound the diffusion near an elliptic equilibrium point of a periodically time-dependent Hamiltonian system. The method is based on the computation of the normal form (up to a certain degree) of that Hamiltonian, in order to obtain an adequate number of (approximate) first integrals of the motion. Then, bounding the variation of those integrals with respect to time provides estimates of the diffusion of the motion.

The example used to illustrate the method is the Elliptic Spatial Restricted Three Body Problem, in a neighbourhood of the points $L_{4,5}$. The mass parameter and the eccentricity are the ones corresponding to the Sun-Jupiter case.
\end{abstract}

\section{Introduction}

The study of the nonlinear stability of an elliptic equilibrium point of a Hamiltonian system is a classical and difficult topic. There are mainly two kind of results concerning this: results of KAM type (perpetual stability on a Cantor set of initial conditions) and results of Nekhoroshev type (stability for an exponentially long time span, on an open set of initial conditions). A survey of both kind of methods can be found in Arnol'd. ${ }^{1}$

In this work we are going to focus on the results of Nekhoroshev type. Our purpose will be to bound the diffusion of the motion near an elliptic equilibrium point of a Hamiltonian system. The kind of methods we are going to use is very similar to the ones used by Giorgilli et al. ${ }^{2}$ and Simó $^{3}$ for autonomous Hamiltonians. 
radius $\rho$ centered at the origin. Let $\eta$ and $T$ be positive numbers, with $\eta<1$.

Definition 1 The origin is $(\eta, T)$-stable iff for all initial condition $z_{0}$ in $B_{\eta \rho}$, the corresponding solution is in the ball $B_{\rho}$ during a time span of lenght $T$.

The values of $\eta$ and $T$ depend on the application we are interested in. For the SunJupiter case, the usual values are $\eta=0.9$ (we allow a diffusion of $10 \%$ ) and $T$ of the order of the age of the Solar system.

In the next sections we are going to see an adapted version of the method used by Simó, ${ }^{3}$ in order to bound the diffusion (near an elliptic equilibrium point) of a timeperiodic Hamiltonian system. For shortness, the methodology will be directly presented on a concrete example. The one used is the study of the nonlinear stability of the $L_{5}$ point of the Elliptic Spatial RTBP (from now on, ESRTBP), taking as eccentricity and mass parameter the ones corresponding to the Sun-Jupiter case, that is, $e=0.048498458$ and $\mu=0.95387536 \times 10^{-3}$.

\section{Expansion of the Hamiltonian}

The first step of the process is to obtain a power expansion of the Hamiltonian around the equilibrium point. The way to obtain that expansion depends strongly on the form of the Hamiltonian we are dealing with. In our example, we have used a recurrence based on the one of the Legendre polynomials.

Now, let us start from the equations of motion of the ESRTBP. They are (see Szebehely ${ }^{4}$ )

$$
\begin{aligned}
x^{\prime \prime}-2 y^{\prime} & =\frac{\partial \omega}{\partial x}, \\
y^{\prime \prime}+2 x^{\prime} & =\frac{\partial \omega}{\partial y}, \\
z^{\prime \prime}+z^{\prime} & =\frac{\partial \omega}{\partial z},
\end{aligned}
$$

where

$$
\omega=\frac{\Omega}{1+e \cos f}, \quad \Omega=\frac{1}{2}\left(x^{2}+y^{2}+z^{2}\right)+\frac{1-\mu}{r_{1}}+\frac{\mu}{r_{2}}+\frac{\mu(1-\mu)}{2},
$$

and $r_{1}^{2}=(x-\mu)^{2}+y^{2}+z^{2}, r_{2}^{2}=(x-\mu+1)^{2}+y^{2}+z^{2}$. Moreover, $e$ is the eccentricity of the two primaries and ' stands for the derivative with respect to the true anomaly $f$.

Defining the momenta $p_{x}=x^{\prime}-y, p_{y}=y^{\prime}+x, p_{z}=z^{\prime}$ and making the (canonic) change of variables $p_{x} \rightarrow p_{x}-\beta, p_{y} \rightarrow p_{y}+\alpha, p_{z} \rightarrow p_{z}, x \rightarrow x+\alpha, y \rightarrow y+\beta$ and $z \rightarrow z$, where $(\alpha, \beta)$ are the $(x, y)$ coordinates of an equilibrium point, the Hamiltonian of the system is

$$
\begin{aligned}
H= & p_{f}+\frac{1}{2}\left(p_{x}^{2}+p_{y}^{2}+p_{z}^{2}\right)+\frac{1}{2}\left(x^{2}+y^{2}+z^{2}\right)+y p_{x}-x p_{y}- \\
& -\frac{1}{1+e \cos f}\left[\frac{1}{2}\left(x^{2}+y^{2}+z^{2}\right)+\alpha x+\beta y+\frac{1-\mu}{r_{P S}}+\frac{\mu}{r_{P J}}\right],
\end{aligned}
$$

where $r_{P S}^{2}=\left(x-x_{S}\right)^{2}+\left(y-y_{S}\right)^{2}+z^{2}, r_{P J}^{2}=\left(x-x_{J}\right)^{2}+\left(y-y_{J}\right)^{2}+z^{2}$, being $\left(x_{S}, y_{S}\right)=(\mu-\alpha,-\beta),\left(x_{J}, y_{J}\right)=(\mu-1-\alpha,-\beta)$. The term $p_{f}$ is the momentum corresponding to $f$, and has been added to obtain an autonomous Hamiltonian. 
¿From now on, we are going to focus on the $L_{5}$ case (the same results will hold for $L_{4}$, due to the symmetry): $x_{S}=1 / 2, y_{S}=\sqrt{3} / 2$.

Our purpose now is to expand this Hamiltonian in a power series of $x, p_{x}, y, p_{y}, z$ and $p_{z}$ :

$$
H\left(x, p_{x}, y, p_{y}, z, p_{z}, f, p_{f}\right)=p_{f}+\sum_{j \geq 2} H_{j}\left(x, p_{x}, y, p_{y}, z, p_{z}, f\right)
$$

where $H_{j}$ is an homogeneouos polynomial of degree $j$, whose coefficients are Fourier series with respect to $f$.

To reduce the global computational effort, we will perform the change of variables that puts $H_{2}$ in normal form, at the same time that the Hamiltonian is expanded. For this reason, the first point we are going to deal with is the normal form of $\mathrm{H}_{2}$.

\subsection{Normal Form of $H_{2}$}

This corresponds to a linear differential equation with periodic coefficients, that can be reduced to constant coefficients by means of the classical Floquet Theorem. We have obtained the change of variables numerically, and we have performed a Fourier analysis in order to obtain an (approximate) analytical expression for the change. At the same time, we want that $H_{2}$ be of the form:

$$
H_{2}=\frac{\omega_{1}}{2}\left(x^{2}+p_{x}^{2}\right)+\frac{\omega_{2}}{2}\left(y^{2}+p_{y}^{2}\right)+\frac{\omega_{3}}{2}\left(z^{2}+p_{z}^{2}\right),
$$

in order to simplify the next steps. This is done by a linear change of variables, that can be composed with the Floquet one to obtain a single change making both things.

Finally, in order to obtain an adequate expression for the calculus of the normal form it is convenient to complexify the expansion. The reason is that, in suitable complex coordinates, $\mathrm{H}_{2}$ takes the form

$$
H_{2}=\sum_{j=1}^{3} \sqrt{-1} \omega_{j} q_{j} p_{j}
$$

that will be very useful (it will save time, memory and the process will not be ill conditioned) during the computations. For that purpose we perform the change of variables

$$
x=\frac{q_{1}+\sqrt{-1} p_{1}}{\sqrt{2}}, \quad p_{x}=\frac{\sqrt{-1} q_{1}+p_{1}}{\sqrt{2}},
$$

and similar expressions for $q_{2}, p_{2}, q_{3}$ and $p_{3}$. The composition of this change with the previous ones provides the final change we were looking for.

\subsection{Expansion}

In order to expand (1), we are going to focus on the expansion of $r_{P S}^{-1}\left(r_{P J}^{-1}\right.$ is obtained with the same procedure). It is known that

$$
\frac{1}{r_{P S}}=\frac{1}{\sqrt{1-2 \rho \cos \psi+\rho^{2}}}=\sum_{n=0}^{\infty} \rho^{n} P_{n}(\cos \psi),
$$

where $\psi$ is the angle between $\left(x_{S}, y_{S}, 0\right)$ and $(x, y, z), \rho^{2}=x^{2}+y^{2}+z^{2}$ and $P_{n}$ is the Legendre polynomial of degree $n$. Let us define $A_{n}$ as $\rho^{n} P_{n}(\cos \psi)$ (note that $A_{n}$ is 


$$
A_{n+1}=\frac{2 n+1}{n+1}\left(x x_{S}+y y_{S}\right) A_{n}-\frac{n}{n+1}\left(x^{2}+y^{2}+z^{2}\right) A_{n-1}
$$

being $A_{0}=1$ and $A_{1}=x x_{S}+y y_{S}$. We have used this recurrence to obtain the expansion of the Hamiltonian, using for $x$ and $y$ the expressions provided by the final "Floquet" change. Finally, the remaining terms of (1) are computed directly.

\section{Normal form}

In what follows, $k$ will be a multiindex, splitted as $k=\left(k^{1}, k^{2}\right)$, where $k^{1}$ and $k^{2}$ correspond to positions and momenta respectively.

The computation of the normal form is based on the following proposition:

Proposition 1 Let us consider the Hamiltonian

$$
H=p_{f}+H_{2}(q, p)+\sum_{i=3}^{r-1} H_{i}(q, p)+H_{r}(q, p, f)+H_{r+1}(q, p, f)+\cdots,
$$

where $r>2, H_{2}(q, p)=\sum \omega_{i} q_{i} p_{i}\left(\omega_{i} \in \mathbb{C}\right), H_{i}(q, p)=\sum_{|k|=i} h_{i}^{k} q^{k^{1}} p^{k^{2}}, H_{r}(q, p, f)=$ $\sum_{|k|=r} h_{r}^{k}(f) q^{k^{1}} p^{k^{2}}$ and $h_{r}^{k}(f)=\sum_{j} h_{r, j}^{k} \exp (j f \sqrt{-1})$. Let us define $G_{r}=G_{r}(q, p, f)=$ $\sum_{|k|=r} g_{r}^{k}(f) q^{k^{1}} p^{k^{2}}$ as follows:

1. if $k^{1} \neq k^{2}$ :

$$
g_{r}^{k}(f)=\frac{c_{k}-h_{r, 0}^{k}}{<\omega, k^{2}-k^{1}>}+\sum_{j \neq 0} \frac{h_{r, j}^{k}}{j \sqrt{-1}-<\omega, k^{2}-k^{1}>} \exp (j f \sqrt{-1}) .
$$

2. if $k^{1}=k^{2}$ :

$$
g_{r}^{k}(f)=\sum_{j \neq 0} \frac{h_{r, j}^{k}}{j \sqrt{-1}} \exp (j f \sqrt{-1}) .
$$

Then, the new Hamiltonian $H^{\prime}$ obtained from $H$ by means of the change of variables given by the generating function $G_{r}$,

$$
H^{\prime}=H+\left\{H, G_{r}\right\}+\frac{1}{2 !}\left\{\left\{H, G_{r}\right\}, G_{r}\right\}+\cdots,
$$

satisfies that

$$
H^{\prime}=p_{f}+H_{2}(q, p)+\sum_{i=3}^{r-1} H_{i}(q, p)+H_{r}^{\prime}(q, p)+H_{r+1}^{\prime}(q, p, f)+\cdots,
$$

where $H_{r}^{\prime}(q, p)=\sum\left(h^{\prime}\right)_{r}^{k} q^{k^{1}} p^{k^{2}}$ and

$$
\left(h^{\prime}\right)_{r}^{k}= \begin{cases}c_{k} & \text { if } k^{1} \neq k^{2} \\ h_{r, 0}^{k} & \text { if } k^{1}=k^{2}\end{cases}
$$

Remark: The value $c_{k}$ that appears in the function $G$ can be selected according to several criteria. In our case, as we want to obtain an integrable normal form, we have chosen that value equal to 0 . It is also possible to chose $c_{k}=h_{r, 0}^{k}$ for the "quasiresonant" values of $k$, to alleviate the effect of the small divisors, but what we obtain in this case is a (in general non-integrable) seminormal form. 

it is not possible to obtain an autonomous normal form, because some of the divisors $j \sqrt{-1}-<\omega, k^{2}-k^{1}>$ appearing in the generating function are zero. In that case, we select the corresponding $g_{r, j}^{k}$ equal to zero.

\subsection{Realification}

The last step of the procedure is to return to real coordinates and to introduce action variables, in order to study the dynamics of the final Hamiltonian. The first part is done by using the inverse of the map given in (2). We denote again by $x, p_{x}$, etc. the real coordinates. The second one by using the Poincaré change $x=\sqrt{2 I_{1}} \cos \varphi_{1}, p_{x}=$ $-\sqrt{2 I_{1}} \sin \varphi_{1}$, and similar for the other variables. In this way, the global transformation preserves the real character of variables and Hamiltonian.

With these changes, we obtain a final Hamiltonian of the form:

$$
H=N+R^{(m+1)}+I_{f}
$$

where $m$ is the degree of the normal form and

$$
N=N\left(I_{1}, I_{2}, I_{3}, \varphi_{3}-f\right),
$$

that depends on $\varphi_{3}-f$ because of the $1: 1$ resonance between the true anomaly $f$ and the angle $\varphi_{3}$ (corresponding to the action $I_{3}$ ) of the vertical mode of the RTBP. Here $I_{f}$ is the actual momentum conjugated to $f$.

All the previous computations have been effectively carried out up to order 20 . The Fourier coefficients which appear in all the process with amplitude less than $10^{-15}$ have been dropped.

\subsection{An approximation to the dynamics}

To study the flow of this Hamiltonian, let us perform the following change of variables

$$
\begin{array}{ccc}
I_{f}=J_{0}-J_{3}, & I_{1}=J_{1}, \quad I_{2}=J_{2}, & I_{3}=J_{3}, \\
f=\psi_{0}, & \varphi_{1}=\psi_{1}, & \varphi_{2}=\psi_{2},
\end{array}
$$

to obtain

$$
H=N+R^{(m+1)}+J_{0}
$$

where

$$
N=N\left(J_{1}, J_{2}, J_{3}, \psi_{3}\right)=\omega_{1} J_{1}+\omega_{2} J_{2}+O_{2}\left(J_{1}, J_{2}, J_{3}\right)
$$

is an integrable Hamiltonian and $R^{(m+1)}=\sum_{j>m} R_{j}$. More concretely, up to second order in the $J_{j}$ variables, $N$ is given by

$$
\begin{aligned}
N_{(2)}= & \omega_{1} J_{1}+\omega_{2} J_{2}+\alpha_{1,1} J_{1}^{2}+\alpha_{1,2} J_{1} J_{2}+\alpha_{2,2} J_{2}^{2}+ \\
& +J_{3}\left[a J_{1}+d J_{2}+\left(b J_{1}+e J_{2}\right) \cos 2 \psi_{3}+\left(c J_{1}+f J_{2}\right) \sin 2 \psi_{3}\right]+g J_{3}^{2},
\end{aligned}
$$

where the numerical values obtained for the constants in the current example are given in Table 1.

The dynamics described by this Hamiltonian is very simple: as $J_{1}$ and $J_{2}$ are first integrals, we can take $J_{1}=C_{1}, J_{2}=C_{2}\left(C_{1,2}\right.$ are constants $)$ to obtain an one-degree 


\begin{tabular}{rrrrrr|}
$\omega_{1}$ & $=$ & $-.8080513430831042 \mathrm{E}-01$ & $\omega_{2}$ & $=$ & $.9967588604945699 \mathrm{E}+00$ \\
$\alpha_{1,1}$ & $=.5842667892951852 \mathrm{E}+00$ & $\alpha_{1,2}$ & $=$ & $-.1551131477053751 \mathrm{E}+00$ \\
$\alpha_{2,2}$ & $=.5641749211325000 \mathrm{E}-02$ & $a$ & $=$ & $.5439031817085827 \mathrm{E}-01$ \\
$b$ & $=.8735695861611382 \mathrm{E}-04$ & $c$ & $=$ & $.2162237916664492 \mathrm{E}-03$ \\
$d$ & $=.5793623535537272 \mathrm{E}-02$ & $e$ & $=$ & $-.5265749230961082 \mathrm{E}-05$ \\
$f$ & $=$ & $-.7753646646134309 \mathrm{E}-05$ & $g$ & $=$ & $-.1787604308798145 \mathrm{E}-03$ \\
\hline
\end{tabular}

of freedom Hamiltonian. In our case this is, essentially, a pendulum depending on the parameters $J_{1}$ and $J_{2}$. Using $N_{(2)}$ as an approximation, the fixed points are located at

$$
\cos 2 \psi_{3}= \pm n / \sqrt{n^{2}+p^{2}}, \quad \sin 2 \psi_{3}= \pm p / \sqrt{n^{2}+p^{2}}, \quad J_{3}=\left(m \pm \sqrt{n^{2}+p^{2}}\right) /(-2 g),
$$

where $m=a J_{1}+d J_{2}, n=b J_{1}+e J_{2}$ and $p=c J_{1}+f J_{2}$. The points with the + sign are hyperbolic and the ones with the - sign are elliptic. If $J_{1}=J_{2}=0$ the angle $\psi_{3}$ is moving quite slowly, with limit frequency equal to zero when $J_{3}$ goes to zero. When $J_{1}$ and/or $J_{2}$ move away from zero a bifurcation occurs. This is related to the 1 to 1 resonance between the vertical mode and the frequency of the elliptic motion. In particular the existence of normally hyperbolic tori of dimensions 2 and 3 follows. However, this will not affect the existence of effective stability, as we shall show.

\section{Bounding the diffusion}

To bound the diffusion speed it is enough to bound $J_{1}^{\prime}, J_{2}^{\prime}$ and $N^{\prime}$, because $N$ is also an approximate first integral of the motion. To do it, we recall that

$$
J_{j}^{\prime}=\left\{J_{j}, H\right\}=\left\{J_{j}, R^{(m+1)}\right\}, \quad j=1,2, \quad N^{\prime}=\left\{N, R^{(m+1)}\right\},
$$

and this can be bounded easily if we have bounds for $\left\|R_{m+1}\right\|$ and for the Poisson bracket.

\subsection{Norms}

The norm used to produce the bounds mentioned above is

$$
\left\|U_{r}\right\|=\sum_{|k|=r}\left\|u_{r}^{k}(f)\right\|=\sum_{|k|=r} \sum_{j}\left|u_{r, j}^{k}\right|
$$

where $U$ is an homogeneous polynomial of degree $r, u_{r}^{k}$ are its coefficients, and $u_{r, j}^{k}$ are the corresponding Fourier coefficients.

This norm has two interesting properties:

1. Let us denote by $\left\|U_{r}\right\|_{B_{\rho}}$ the sup norm of the homogeneous polynomial $U_{r}$ of degree $r$ over the ball centered in the origin and with radius $\rho$, and for all (real) time $f$. Then

$$
\left\|U_{r}\right\|_{B_{\rho}} \leq\left\|U_{r}\right\| \rho^{r} .
$$

2. Let $U_{r}$ and $V_{s}$ be homogeneous polynomials of degree $r$ and $s$. Then

$$
\left\|\left\{U_{r}, V_{s}\right\}\right\| \leq r s\left\|U_{r}\right\|\left\|V_{s}\right\| .
$$


obtained using different norms (see Simó ${ }^{3}$ ).

\subsection{Final results}

¿From (4), and using the norms mentioned above, it is not difficult to obtain

$$
\left|J_{j}^{\prime}\right| \leq \sum_{l>m} l\left\|R_{l}\right\| \rho^{l}, \quad j=1,2,
$$

where the values $\left\|R_{l}\right\|$ can be estimated of the following form: ¿From the recurrence (3), we obtain bounds for the norms of the homogeneous polynomials $H_{r}$ that appear in the Hamiltonian expansion. Then, we bound the norms of the homogeneous components of the successive Hamiltonians obtained (using $G_{3}$ to $G_{20}$ ). In particular we get $\left\|R_{l}\right\|$. We have used the recurrent relations given by the Lie transforms to obtain the bounds up to order 800 . Note that, with this, we have bounded the diffusion with respect to the planar variables.

Now we would like to bound the diffusion in the vertical direction. To do that, instead of bounding $\left|N^{\prime}\right|$, let us define first $\widetilde{N}$ as $N$ minus the part of $N$ depending only on $J_{1}, J_{2}$ (obviously, $\widetilde{N}$ is also an approximate first integral).

Now, we bound $\widetilde{N}^{\prime}$ (as it was done with $J_{1,2}^{\prime}$ by means of (4)). From this bound we need to derive a bound for the diffusion in the vertical direction. At this point we want to remark a difficulty in this approach: $J_{3}$ is not an approximate first integral but a fast variable of the system. The $J_{3}$ variable can oscillate around the elliptic equilibrium point mentioned in Section 3.3.

We have used the following approach: to ensure that if we start at a ball of radius $\eta \rho$ then we end, at most, at the boundary of a ball of radius $\rho$ we should have

$$
\min _{\left(z_{i}, z_{f}\right) \in \partial B_{\eta \rho} \times \partial B_{\rho}}\left|\widetilde{N}\left(z_{f}\right)-\widetilde{N}\left(z_{i}\right)\right| \geq T\left|\widetilde{N}^{\prime}\right|_{B_{\rho}}
$$

We have used $\eta=0.5$ (see later) and $T$ the approximate age of the Solar system $\left(T=4.5 \times 10^{9}\right.$ years $\approx 2.4 \times 10^{9}$ adimensional units $)$. The maximum value of $\rho$ satisfying the previous condition is then obtained.

Notice the following fact about this method: even if the diffusion for $\widetilde{N}$ were exactly 0 , we would have "diffusion" in the vertical direction, due to the (possible) libration of $J_{3}$. Obviously, this diffusion is not a real one. Otherwise, this "false" diffusion is a fast phenomenon that can be observed, and it has a physical meaning.

To have an idea of the amount of this diffusion, we can compute from the normal form a bound $A$ of the maximum size of the above-mentioned libration motion. This size depends on the value $J_{3}^{*}$ for which we have the elliptic equilibrium point (see Section 3.3). It is not difficult to obtain that $A / J_{3}^{*}<0.27$ (this follows, essentially, from a careful analysis of $\left.N_{(2)}\right)$. This is the reason why we have taken a small value of $\eta(0.5$ instead of something close to 1 ).

Finally, using $\eta=0.5$ and $T=2.4 \times 10^{9}$, as stated before, the radius of the ball of $(\eta, T)$-stability is found to be $\rho=0.571 \times 10^{-3}$.

Now we need to send that ball back to the original coordinates. We are going to split this process in two steps: the normal form change (the nonlinear part of the change obtained in Section 3) and the Floquet change.

The deformation produced by the normal form change can be bounded from the norm of the coefficients of this change of variables. This shows that this ball could be reduced in a factor of $5 / 6$, that is, to the value $\hat{\rho}=0.476 \times 10^{-3}$. 
on the true anomaly $f$ in a periodic way. To obtain a region of effective stability independent of $f$ we have intersected (for all $f$ ) all these regions. This produces a domain with a "banana" shape, having a minimum diameter of $0.11 \hat{\rho}$ and a maximum one of 9.8 $\hat{\rho}$. This implies that the largest ball contained in that domain and centered at the origin $\left(L_{4,5}\right)$ has a radius of $0.52 \times 10^{-4}$ adimensional units. On the other hand, in some "good" direction, the distance of stability is multiplied by a factor of $9.8 / 0.11 \approx 89$.

\subsection{Remarks}

Numerical simulations of the motion close to $L_{4,5}$ in the same problem show that there exist points close to $L_{4,5}$ (but outside the region mentioned before) that go away in a very short time (see Gómez et al. ${ }^{5} ;$ Simó $^{6}$ ). This is due to the effect of the 1:1 resonance between the true anomaly and the vertical mode of the RTBP (this phenomenon is now being studied (see Jorba and Simón ${ }^{7}$ )). This effect is more evident when the value of $\mu$ is bigger (i.e. Earth-Moon system), because the (angle of) splitting between the stable and unstable manifolds of the hyperbolic tori mentioned above grows very fast when $\mu$ increases.

There are also other regions of stability that are even larger, allowing for large values for the inclinations (see Gómez et al. ${ }^{5}$ ). These regions are the ones containing the Trojan asteroids.

Acknowledgements. The authors have been partially supported by the CICYT grant ESP91-0403.

\section{References}

1. V.I. Arnol'd: "Dynamical Systems III”, Springer-Verlag (1988).

2. A. Giorgilli, A. Delshams, E. Fontich, L. Galgani and C. Simó: Effective stability for a Hamiltonian system near an elliptic equilibrium point, with an application to the Restricted Three Body Problem, Journal of Differential Equations 77:1 (1989).

3. C. Simó: Estabilitat de sistemes Hamiltonians, Mem. de la Real Acad. de Cienc. i Art. de Barcelona, Vol. XLVIII, no. 7 (1989).

4. V. Szebehely: "Theory of Orbits", Academic Press (1967).

5. G. Gómez, A. Jorba, J. Masdemont and C. Simó: Study of Poincaré maps for Orbits near Lagrangian Points, ESOC contract 9711/91/D/IM(SC), Second Progress Report (1992).

6. C. Simó: Stability regions for the elliptic RTBP near the triangular points (in progress).

7. A. Jorba and C. Simó: Hyperbolic tori close to the equilateral points of the elliptic RTBP (in progress). 